\title{
一个捕食椭圆型方程组正解的存在性、唯一 性与稳定性
}

王明新 (1) (2)*, 王旭勃 (3)

(1) 东南大学数学系, 南京 210018

(2) 徐州师范大学数学系, 徐州 221116

(3) Division of Natural Science and Mathematics, Macon State College, Macon, GA 31206, USA

*E-mail: mxwang@seu.edu.cn

收稿日期: 2007-05-14; 接受日期: 2008-08-02

国家自然科学基金 (批准号: 10471022, 10771032) 和江苏省基础研究计划 (批准号: BK2006088) 资助项目

摘要 研究一个修正的 Leslie-Gower 和 Holling-Type II 型的捕食模型的椭圆型方程组 的齐次 Dirichlet 边值问题的正解. 给出了存在性、唯一性、不存在性、分支和稳定性. 特别 地, 得到了连接半平凡解和极限方程的唯一正解的连通分支.

关键词捕食模型 正解 分支 存在性和唯一性 稳定性

$\operatorname{MSC}(2000)$ 主题分类 $35 \mathrm{~J} 55,35 \mathrm{~B} 25,92 \mathrm{C} 25$

\section{1 引言}

本文研究椭圆型方程组的边值问题

$$
\left\{\begin{array}{lll}
-d_{1} \Delta u=u\left(a-u-\frac{m v}{k_{1}+u}\right), & x \in \Omega ; & \left.u\right|_{\partial \Omega}=0 \\
-d_{2} \Delta v=v\left(b-\frac{c v}{k_{2}+u}\right), & x \in \Omega ; & \left.v\right|_{\partial \Omega}=0
\end{array}\right.
$$

的正解, 其中 $\Omega \subset \mathbb{R}^{n}$ 是一个带有光滑边界 $\partial \Omega$ 的有界区域, 正常数 $d_{1}, d_{2}$ 是扩散系数. 它对 应的常微分方程, 作为修正的 Leslie-Gower 和 Holling-Type II 型捕食模型由 Aziz-Alaoui 和 Okiye 提出 ${ }^{[1]}$. 在该模型中, $u$ 和 $v$ 分别表示食物和猎物的分布密度, $a, m, k_{1}, b, c$ 和 $k_{2}$ 都是 正常数, 其生物学意义可参见文献 [1].

做尺度变换, 不妨认为 $d_{1}=d_{2}=1$. 不失一般性, 我们取 $c=k_{2}=1$, 再把 $k_{1}$ 记成 $k$. 这 样, 所要讨论的问题就成为

$$
\left\{\begin{array}{lll}
-\Delta u=u\left(a-u-\frac{m v}{k+u}\right), & x \in \Omega ; & \left.u\right|_{\partial \Omega}=0, \\
-\Delta v=v\left(b-\frac{v}{1+u}\right), & x \in \Omega ; & \left.v\right|_{\partial \Omega}=0 .
\end{array}\right.
$$


先给出一些预备知识. 令 $\lambda_{1}(q)<\lambda_{2}(q) \leqslant \lambda_{3}(q) \leqslant \cdots$ 是特征值问题

$$
-\Delta w+q(x) w=\lambda w, \quad x \in \Omega ;\left.\quad w\right|_{\partial \Omega}=0
$$

的全部特征值, 其中 $q(x) \in C(\bar{\Omega}) . \lambda_{1}(q)$ 是简单的, 并且当 $q_{1}(x) \leqslant q_{2}(x), q_{1}(x) \not \equiv q_{2}(x)$ 时, 还有 $\lambda_{i}\left(q_{1}\right)<\lambda_{i}\left(q_{2}\right)$. 记 $\lambda_{i}=\lambda_{i}(0)$. 考虑边值问题

$$
-\Delta w+q(x) w=a w-f(x) w^{2}, \quad x \in \Omega ;\left.\quad w\right|_{\partial \Omega}=0,
$$

其中 $f(x)$ 是 $\bar{\Omega}$ 上的正的连续函数. 由文献 $[2,3]$ 知, 如果 $a \leqslant \lambda_{1}(q)$, 那么 $u=0$ 是 (1.2) 式 的唯一非负解, 而当 $a>\lambda_{1}(q)$ 时, (1.2) 式有唯一正解. 当 $q=0, f=1, a>\lambda_{1}$ 时, 把这个唯 一正解记成 $\theta_{a}$. 记 $C_{0}(\bar{\Omega})=\left\{u \in C(\bar{\Omega}):\left.u\right|_{\partial \Omega}=0\right\}$, 并定义算子 $L_{a}$ :

$$
L_{a} w=-\Delta w+\left(2 \theta_{a}-a\right) w, \quad w \in C^{2}(\Omega) \cap C_{0}(\bar{\Omega}) .
$$

由文献 $[2,3]$ 知, $L_{a}$ 的所有特征值都是正的.

引理 1.1 (i) 设 $(u, v)$ 是问题 (1.1) 的非负解, 则有

$$
u(x)<a, \quad v(x)<b(1+a), \quad \forall x \in \bar{\Omega} .
$$

(ii) 如果问题 (1.1) 有正解 $(u, v)$, 则 $a, b>\lambda_{1}$ 并且

$$
u(x)<\theta_{a}(x)<a, \quad \theta_{b}(x)<v(x)<\theta_{a, b}(x)<b(1+a), \quad \forall x \in \Omega,
$$

这里 $\theta_{a, b}(x)$ 是问题

$$
-\Delta u=b u-\frac{u^{2}}{1+\theta_{a}(x)}, \quad x \in \Omega ;\left.\quad u\right|_{\partial \Omega}=0
$$

的唯一正解. 由此知, 如果问题 (1.1) 有正解, 那么 $b>\lambda_{1}, a>\lambda_{1}\left(\frac{m \theta_{b}}{k+\theta_{a}}\right)$.

证明 利用最大值原理可以直接推出结论 (i).

(ii) 如果问题 $(1.1)$ 有正解 $(u, v)$, 则

$$
-\Delta u=u\left(a-u-\frac{m v}{k+u}\right) \leqslant u(a-u)<a u, \quad x \in \Omega ;\left.\quad u\right|_{\partial \Omega}=0 .
$$

由此推出 $a>\lambda_{1}$. 再由唯一性知 $u<\theta_{a}$. 其余部分同理可证.

\section{2 存在性和不存在性}

本节讨论正解的存在性和非存在性. 首先叙述雉上的不动点指数的若干结果 ${ }^{[4]}$. 相关内 容和应用, 还可以参见文献 $[5-15]$.

记 $\mathbf{u}=(u, v), X=C_{0}(\bar{\Omega}) \oplus C_{0}(\bar{\Omega}), Y=C(\bar{\Omega}) \oplus C(\bar{\Omega}), Z=C^{2}(\bar{\Omega}) \oplus C^{2}(\bar{\Omega}), W=\{\mathbf{u} \in$ $X: u, v \geqslant 0, x \in \Omega\}$ 是 $X$ 中的正雉. 设 $\mathcal{O}$ 是 $W$ 中的开集. 对于 $\mathbf{z} \in \mathcal{O}$, 定义楔

$$
W_{\mathbf{z}}=\operatorname{closure}\{\mathbf{u} \in X: \text { 存在 } s>0 \text { 使得 } \mathbf{z}+s \mathbf{u} \in W\} \text {. }
$$

取 $X_{\mathbf{z}}$ 是 $X$ 的包含在 $W_{\mathbf{z}}$ 中的最大子空间. 设 $K: \mathcal{O} \rightarrow W$ 是紧算子并且 Fréchet 可微. 若 存在 $X$ 的子空间 $Y_{\mathbf{z}}$, 使得 $X=X_{\mathbf{z}} \oplus Y_{\mathbf{z}}$, 则算子 $K$ 在 $\mathbf{z}$ 处的指数 $\operatorname{Ind}_{W}(K, \mathbf{z})$ 可以通过研 究它在空间 $X_{\mathbf{z}}$ 和 $Y_{\mathbf{z}}$ 中的特征值问题而得到.

设 $T: X \rightarrow Y_{\mathbf{z}}$ 是投影算子. 如果 $K^{\prime}(\mathbf{z})$ 在 $W_{\mathbf{z}}$ 内没有非零的不动点, 那么指数 $\operatorname{Ind}_{W}(K, \mathbf{z})$ 有定义. 同时, $\operatorname{Ind}_{W}(K, \mathbf{z})=0$ 如果 $T \circ K^{\prime}(\mathbf{z})$ 在 $W_{\mathbf{z}}$ 内有一个大于 1 的特 征值, $\operatorname{Ind}_{W}(K, \mathbf{z})=\operatorname{Ind}_{X_{\mathbf{z}}}\left(K^{\prime}(\mathbf{z}), \mathbf{0}\right)=(-1)^{r}$ 如果 $T \circ K^{\prime}(\mathbf{z})$ 没有这样的特征值, 这里 $\operatorname{Ind}_{X_{\mathbf{z}}}\left(K^{\prime}(\mathbf{z}), \mathbf{0}\right)$ 是在空间 $X_{\mathbf{z}}$ 中算子 $K^{\prime}(\mathbf{z})$ 在点 $\mathbf{0}$ 处的不动点指数, $r$ 是算子 $K^{\prime}(\mathbf{z})$ 在空 间 $X_{\mathbf{z}}$ 中所有大于 1 的特征值的重数之和. 见文献 [4] 的定理 2.2 和 2.3 . 
按如下方式定义集合 $\mathcal{O}$ 和算子 $K$ :

$$
\begin{aligned}
& \mathcal{O}=\{\mathbf{u} \in W: 0 \leqslant u<a, \quad 0 \leqslant v<b(1+a), \quad x \in \bar{\Omega}\}, \\
& K(\mathbf{u})=(-\Delta+M)^{-1}\left(M u+u\left(a-u-\frac{m v}{k+u}\right), M v+v\left(b-\frac{v}{1+u}\right)\right),
\end{aligned}
$$

这里 $M$ 是一个待定的大常数. 显然, $\mathcal{O}$ 包含问题 (1.1) 的所有非负解, $K: \mathcal{O} \rightarrow W$ 是可微的 紧算子. 研究问题 (1.1) 的正解等价于研究算子 $K$ 的正不动点.

容易看出, $(0,0),\left(\theta_{a}, 0\right)$ 和 $\left(0, \theta_{b}\right)$ 是问题 $(1.1)$ 的所有可能的平凡和半平凡非负解 (如果 $\left.a, b>\lambda_{1}\right)$. 记 $\mathbf{0}=(0,0), \mathbf{u}^{*}=\left(\theta_{a}, 0\right), \mathbf{v}^{*}=\left(0, \theta_{b}\right)$.

命题 2.1 假设 $a \neq \lambda_{1}, b \neq \lambda_{1}$.

(i) $\operatorname{Ind}_{W}(K, \mathbf{0})=0$ 如果 $a>\lambda_{1}$ 或者 $b>\lambda_{1}, \operatorname{Ind}_{W}(K, \mathbf{0})=1$ 如果 $a, b<\lambda_{1}$.

(ii) 假设 $b>\lambda_{1}, a \neq \lambda_{1}\left(\frac{m}{k} \theta_{b}\right)$. 则当 $a>\lambda_{1}\left(\frac{m}{k} \theta_{b}\right)$ 时 $\operatorname{Ind}_{W}\left(K, \mathbf{v}^{*}\right)=0$, 当 $a<\lambda_{1}\left(\frac{m}{k} \theta_{b}\right)$ 时 $\operatorname{Ind}_{W}\left(K, \mathbf{v}^{*}\right)=1$.

(iii) 假设 $a>\lambda_{1}$. 则当 $b>\lambda_{1}$ 时 $\operatorname{Ind}_{W}\left(K, \mathbf{u}^{*}\right)=0$, 当 $b<\lambda_{1}$ 时 $\operatorname{Ind}_{W}\left(K, \mathbf{u}^{*}\right)=1$.

证明 设 $\varphi_{1}$ 是对应于 $\lambda_{1}$ 的特征函数, 则 $\varphi_{1}>0$.

(i) 取 $\mathbf{z}=\mathbf{0}$. 直接计算知 $W_{\mathbf{0}}=W, X_{\mathbf{0}}=\{\mathbf{0}\}, Y_{\mathbf{0}}=X, T=I$, 其中 $I$ 是 $X$ 上的单位算 子. 易证

$$
K^{\prime}(\mathbf{0}) \mathbf{u}=(-\Delta+M)^{-1}((a+M) u,(b+M) v),
$$

并且 $K^{\prime}(\mathbf{0})$ 在 $W_{\mathbf{0}}$ 中没有非零不动点. 同时, 其对应的特征向量属于 $W_{\mathbf{0}}$ 的算子 $K^{\prime}(\mathbf{0})$ 的特 征值是 $\lambda_{a}=\frac{a+M}{\lambda_{1}+M}, \lambda_{b}=\frac{b+M}{\lambda_{1}+M}$. 如果 $a>\lambda_{1}$ 或者 $b>\lambda_{1}$, 那么 $\lambda_{a}>1$ 或者 $\lambda_{b}>1$, 于是 $\operatorname{Ind}_{W}(K, \mathbf{0})=0$. 如果 $a, b<\lambda_{1}$, 那么 $\lambda_{a}, \lambda_{b}<1$, 于是 $\operatorname{Ind}_{W}(K, \mathbf{0})=\operatorname{Ind}_{X_{\mathbf{0}}}\left(K^{\prime}(\mathbf{0}), \mathbf{0}\right)=(-1)^{r}$. 因为 $X_{\mathbf{0}}=\{\mathbf{0}\}$, 所以 $r=0$. 故 $\operatorname{Ind}_{W}(K, \mathbf{0})=1$.

(ii) 取 $\mathbf{z}=\mathbf{v}^{*}$. 简单计算得 $W_{\mathbf{v}^{*}}=\{\mathbf{u} \in X: u \geqslant 0\}, X_{\mathbf{v}^{*}}=\{\mathbf{u} \in X: u=0\}$. 令 $Y_{\mathbf{v}^{*}}=\{\mathbf{u} \in X: v=0\}$, 则 $X=X_{\mathbf{v}^{*}} \oplus Y_{\mathbf{v}^{*}}, T:(u, v) \rightarrow(u, 0)$. 同时还有

$$
K^{\prime}\left(\mathbf{v}^{*}\right) \mathbf{u}=(-\Delta+M)^{-1}\left(\left(a+M-\frac{m}{k} \theta_{b}\right) u,\left(b+M-2 \theta_{b}\right) v+\theta_{b}^{2} u\right) .
$$

这里, 取 $M$ 适当大, 使得 $a+M-\frac{m}{k} \theta_{b}>0, b+M-2 \theta_{b}>0$ 在 $\bar{\Omega}$ 上成立. 由于 $a \neq \lambda_{1}\left(\frac{m}{k} \theta_{b}\right)$, 并且 $L_{b} w=0$ 没有非平凡解, 所以 $K^{\prime}\left(\mathbf{v}^{*}\right)$ 在 $W_{\mathbf{v}^{*}} \backslash\{\mathbf{0}\}$ 中没有不动点, 因此 $\operatorname{Ind}_{W}\left(K, \mathbf{v}^{*}\right)$ 存 在.

下面讨论 $T \circ K^{\prime}\left(\mathbf{v}^{*}\right)$ 的特征值. 根据投影算子 $T:(u, v) \rightarrow(u, 0)$ 的形式知, $T \circ K^{\prime}\left(\mathbf{v}^{*}\right)$ 的特征向量都是 $(u, 0)$ 的形式. 容易看出, $\lambda$ 是 $T \circ K^{\prime}\left(\mathbf{v}^{*}\right)$ 的一个特征值, $(u, 0)$ 是对应的特 征向量, 当且仅当 $u$ 是问题

的解. 由此知 $\lambda \neq 0$. 于是

$$
\lambda u=(-\Delta+M)^{-1}\left(a+M-\frac{m}{k} \theta_{b}\right) u, \quad u \neq 0
$$

$$
\left\{\begin{array}{l}
-\Delta u+\frac{m \theta_{b}}{k} u+\frac{\lambda-1}{\lambda}\left(a+M-\frac{m \theta_{b}}{k}\right) u=a u, \quad x \in \Omega, \\
\left.u\right|_{\partial \Omega}=0,\left.\quad u\right|_{\Omega} \neq \equiv 0 .
\end{array}\right.
$$

因此, 存在 $j \geqslant 1$, 使得

$$
a=\lambda_{j}\left(\frac{m \theta_{b}}{k}+\frac{\lambda-1}{\lambda}\left(a+M-\frac{m \theta_{b}}{k}\right)\right) .
$$


当 $a>\lambda_{1}\left(\frac{m}{k} \theta_{b}\right)$ 时, 容易看出, 存在 $\lambda>1$, 使得 (2.2) 式对于 $j=1$ 成立. 所以 $\operatorname{Ind}_{W}\left(K, \mathbf{v}^{*}\right)=0$.

当 $a<\lambda_{1}\left(\frac{m}{k} \theta_{b}\right)$ 时, 如果 $(2.2)$ 式成立, 那么 $\lambda<1$ 并且 $\operatorname{Ind}_{W}\left(K, \mathbf{v}^{*}\right)=\operatorname{Ind}_{X_{\mathbf{v}^{*}}}\left(K^{\prime}\left(\mathbf{v}^{*}\right), \mathbf{0}\right)$ $=(-1)^{r}$. 下面计算 $r$. 假设 $\mu$ 是 $K^{\prime}\left(\mathbf{v}^{*}\right)$ 的一个特征值, $(\xi, \zeta) \in X_{\mathbf{v}^{*}}$ 是对应的特征向量, 则 $\xi=0, \zeta$ 是 $\mu \zeta=(-\Delta+M)^{-1}\left(b+M-2 \theta_{b}\right) \zeta$ 的一个非零解. 因此

$$
-\Delta \zeta+2 \theta_{b} \zeta+\frac{\mu-1}{\mu}\left(b+M-2 \theta_{b}\right) \zeta=b \zeta, \quad x \in \Omega ; \quad \zeta=0, \quad x \in \partial \Omega .
$$

于是, 存在 $j \geqslant 1$, 使得 $b=\lambda_{j}\left(2 \theta_{b}+\frac{\mu-1}{\mu}\left(b+M-2 \theta_{b}\right)\right)$. 因为 $\lambda_{1}\left(2 \theta_{b}\right)>b$, 所以 $\mu<1$. 故 $r=0, \operatorname{Ind}_{W}\left(K, \mathbf{v}^{*}\right)=1$.

结论 (iii) 的证明类似. 证毕.

定理 2.1 若 $b>\lambda_{1}, a>\lambda_{1}\left(\frac{m}{k} \theta_{b}\right)$, 则问题 (1.1) 至少有一个正解.

证明 先证明 $\operatorname{Ind}_{W}(K, \mathcal{O})=1$. 为此, 对于 $\mu \in[0,1]$, 定义紧算子 $K_{\mu}: X \rightarrow X:$

$$
K_{\mu}(\mathbf{u})=(-\Delta+M)^{-1}\left(M u+\mu\left(a u-u^{2}-\frac{m u v}{k+u}\right), M v+\mu\left(b v-\frac{v^{2}}{1+u}\right)\right) .
$$

如果存在 $\mathbf{u} \in W, \mu \in[0,1]$, 使得 $K_{\mu}(\mathbf{u})=\mathbf{u}$, 则由最大值原理得 $\mathbf{u} \in \mathcal{O}$, 因此, 对所有 的 $\mu \in[0,1], K_{\mu}$ 在 $\partial \mathcal{O}$ 上没有不动点. 再由同伦不变性知 $\operatorname{Ind}_{W}(K, \mathcal{O})=\operatorname{Ind}_{W}\left(K_{1}, \mathcal{O}\right)=$ $\operatorname{Ind}_{W}\left(K_{0}, \mathcal{O}\right)$. 由于 $K_{0}$ 只有平凡的不动点 $(0,0)$, 故 $\operatorname{Ind}_{W}\left(K_{0}, \mathcal{O}\right)=\operatorname{Ind}_{W}\left(K_{0}, \mathbf{0}\right)$. 同于 命题 2.1 中关于 $\mathbf{z}=\mathbf{0}$ 的讨论, $\operatorname{Ind}_{W}\left(K_{0}, \mathbf{0}\right)=1$, 从而 $\operatorname{Ind}_{W}(K, \mathcal{O})=1$.

反证. 如果 $K$ 在 $\mathcal{O}$ 中没有不动点, 因为 $b>\lambda_{1}, a>\lambda_{1}\left(\frac{m}{k} \theta_{b}\right)$, 利用命题 2.1 和度的可加 性得

$$
0=\operatorname{Ind}_{W}(K, \mathbf{0})+\operatorname{Ind}_{W}\left(K, \mathbf{u}^{*}\right)+\operatorname{Ind}_{W}\left(K, \mathbf{v}^{*}\right)=\operatorname{Ind}_{W}(K, \mathcal{O})=1,
$$

这是一个矛盾. 故问题 (1.1) 至少有一个正解. 证毕.

定理 2.2 假设 $m, b$ 固定并且 $b>\lambda_{1}$.

(i) 记 $\mathcal{A}=\left\{\mathbf{0}, \mathbf{u}^{*}, \mathbf{v}^{*}\right\}$. 如果 $a<\lambda_{1}\left(\frac{m}{k} \theta_{b}\right)$, 则存在正常数 $\delta=\delta(a, k)$, 使得问题 (1.1) 在 $\mathcal{A}$ 的 $\delta$ 邻域 $\mathcal{A}_{\delta}$ 内无正解.

(ii) 存在满足 $\tilde{k}^{2}<m b$ 的非负常数 $\tilde{k}$, 使得当 $k>\tilde{k}, a \leqslant \lambda_{1}\left(\frac{m}{k} \theta_{b}\right)$ 时, 问题(1.1)无正解.

定理 2.1 和 2.2 的一个直接推论是: 如果 $k>\tilde{k}$, 那么问题 (1.1) 有正解当且仅当 $b>\lambda_{1}$, $a>\lambda_{1}\left(\frac{m}{k} \theta_{b}\right)$.

定理 2.2 的证明 (i) 若 $\mathbf{u}$ 是问题 (1.1) 的正解, 则 $v>\theta_{b}$. 由椭圆型方程的正则性理 论知, 问题 (1.1) 的所有正解构成一个紧集. 因此, 存在 $\delta_{1}>0$, 使得 $\|\mathbf{u}\| \geqslant \delta_{1},\left\|\mathbf{u}-\mathbf{u}^{*}\right\| \geqslant \delta_{1}$ 对问题 (1.1) 的任一正解 $\mathbf{u}$ 都成立.

下面利用隐函数定理证明, 在 $\mathbf{v}^{*}$ 的邻域内问题 (1.1) 没有异于 $\mathbf{v}^{*}$ 的解. 对于 $\mathbf{u} \in X \cap Z$, 定义

$$
\mathcal{F}(a ; \mathbf{u})=\left(\Delta u+a u-u^{2}-\frac{m u v}{k+u}, \Delta v+b v-\frac{v^{2}}{1+u}\right) .
$$

则问题 $(1.1)$ 等价于 $\mathcal{F}(a ; \mathbf{u})=0$. 易知

$$
\mathcal{F}\left(a ; \mathbf{v}^{*}\right)=0, \quad \mathcal{F}_{\mathbf{u}}\left(a ; \mathbf{v}^{*}\right) \mathbf{u}=\left(\Delta u+a u-\frac{m \theta_{b}}{k} u, \Delta v+b v-2 \theta_{b} v+\theta_{b}^{2} u\right) .
$$

如果 $\mathcal{F}_{\mathbf{u}}\left(a ; \mathbf{v}^{*}\right) \mathbf{u}=0$, 由于 $a<\lambda_{1}\left(\frac{m}{k} \theta_{b}\right)$, 所以 $u=0$, 从而 $v=0$, 因此 $\mathcal{F}_{\mathbf{u}}\left(a ; \mathbf{v}^{*}\right)$ 是可逆的. 由 Fredholm 二择一性质和椭圆型方程的正则性知, $\mathcal{F}_{\mathbf{u}}\left(a ; \mathbf{v}^{*}\right)$ 是满射, 故 $\mathcal{F}_{\mathbf{u}}\left(a ; \mathbf{v}^{*}\right)$ 满单射. 根据隐函数定理, 在 $\mathbf{v}^{*}$ 的邻域内, 问题 (1.1) 有唯一解 $\mathbf{v}^{*}$. 
(ii) 由于 $\theta_{b}<b$, 故存在 $\varepsilon_{0}>0$, 使得 $\theta_{b}<b-\varepsilon_{0}$. 如果 $\mathbf{u}$ 是问题 (1.1) 的正解, 则 $v>\theta_{b}$ 并且 $a=\lambda_{1}\left(u+\frac{m v}{k+u}\right)>\lambda_{1}\left(u+\frac{m \theta_{b}}{k+u}\right)$. 利用 $\theta_{b}<b-\varepsilon_{0}$ 易证, 如果 $k^{2} \geqslant m\left(b-\varepsilon_{0}\right)$, 则 $\frac{m \theta_{b}}{k} \leqslant u+\frac{m \theta_{b}}{k+u}$, 从而 $a>\lambda_{1}\left(\frac{m}{k} \theta_{b}\right)$. 这说明, 如果 $k^{2} \geqslant m\left(b-\varepsilon_{0}\right)$, 则对所有的 $a \leqslant \lambda_{1}\left(\frac{m}{k} \theta_{b}\right)$, 问题 (1.1) 没有正解. 定义集合

$$
\mathcal{B}=\left\{k^{*}>0: \text { 问题 (1.1) 对所有 } k \geqslant k^{*}, a \leqslant \lambda_{1}\left(\frac{m}{k} \theta_{b}\right) \text { 没有正解 }\right\} \text {, }
$$

则 $\left[m\left(b-\varepsilon_{0}\right)\right]^{1 / 2} \in \mathcal{B}$. 取 $\tilde{k}=\inf \mathcal{B}$, 则结论成立. 证毕.

注 2.1 固定 $a, m, k$ 且 $a>\lambda_{1}$. 因为当 $b \searrow \lambda_{1}$ 时, $\theta_{b} \rightarrow 0$ 在 $\bar{\Omega}$ 上一致成立, 当 $b \rightarrow \infty$ 时, $\theta_{b} \rightarrow \infty$ 在 $\Omega$ 的任一紧集上一致成立 ${ }^{[10]}$, 所以函数 $g(b):=\lambda_{1}\left(\frac{m \theta_{b}}{k+a}\right)$ 是 $b$ 的严格增函数, 并且满足 $g\left(\lambda_{1}\right)=\lambda_{1}<a, g(\infty)=\infty$. 于是, 存在 $b^{*} \in\left(\lambda_{1}, \infty\right)$, 使得当 $b>b^{*}$ 时, 问题 (1.1) 没有正解 (引理 1.1).

\section{3 分支、唯一性和稳定性}

在第 2 节中, 我们利用度理论研究了当 $a>\lambda_{1}\left(\frac{m}{k} \theta_{b}\right), b>\lambda_{1}$ 时, 问题 (1.1) 的正解的存 在性. 本节研究正解的分支、唯一性与稳定性. 先证一个引理.

引理 3.1 假设 $a_{i}^{*} \rightarrow a^{*}$, 问题 (1.1) 当 $a=a_{i}^{*}$ 时有正解 $\left(u_{i}, v_{i}\right)$. 如果在空间 $C(\bar{\Omega})$ 中 $u_{i} \rightarrow u$, 并且存在 $x_{0} \in \Omega$, 使得 $u\left(x_{0}\right)=0$, 则 $u(x) \equiv 0, v_{i} \rightarrow \theta_{b}, a^{*}=\lambda_{1}\left(\frac{m}{k} \theta_{b}\right)$.

证明 容易看出, $u(x)$ 是非负的. 利用估计式 (1.4) 和椭圆型方程的正则性可知, 存在 $v$, 使得在空间 $\left[C^{2}(\Omega) \cap C(\bar{\Omega})\right]^{2}$ 中 $\left(u_{i}, v_{i}\right) \rightarrow(u, v)$, 并且 $(u, v)$ 满足 $a=a^{*}$ 时的问题 (1.1). 运 用强最大值原理于问题 (1.1) 的第 1 个方程, 得 $u(x) \equiv 0$, 从而 $v=\theta_{b}$. 令 $\hat{u}_{i}=\frac{u_{i}}{\left\|u_{i}\right\|_{\infty}}$, 则在 空间 $C^{2}(\Omega) \cap C(\bar{\Omega})$ 中 $\hat{u}_{i} \rightarrow \hat{u}$, 并且 $\hat{u}$ 是问题

$$
-\Delta \hat{u}=\left(a^{*}-\frac{m}{k} \theta_{b}\right) \hat{u}, \quad x \in \Omega ;\left.\quad \hat{u}\right|_{\partial \Omega}=0
$$

的正解, 故 $a^{*}=\lambda_{1}\left(\frac{m}{k} \theta_{b}\right)$. 证毕.

定理 3.1 (整体分支) 设 $m, k, b$ 固定且 $b>\lambda_{1}$. 把 $a$ 看成分支参数, 并记 $a_{1}=\lambda_{1}\left(\frac{m}{k} \theta_{b}\right)$, 则在 $a-\mathbf{u}$ 平面上存在连接 $\left(a_{1}, \mathbf{v}^{*}\right)$ 和 $(\infty, \mathbf{u}(\infty))$ 的问题 (1.1) 的正解的连通分支.

证明 取 $K(\mathbf{u})$ 如 $(2.1)$ 式定义, 并把问题 (1.1) 等价地写成 $\mathbf{F}(a ; \mathbf{u}): \equiv K(\mathbf{u})-\mathbf{u}=0$, $\mathbf{u} \in Z \cap X$. 同于第 2 节,

$$
\begin{aligned}
& \mathbf{F}_{\mathbf{u}}\left(a ; \mathbf{v}^{*}\right) \mathbf{u}=(-\Delta+M)^{-1}\left(\left(a+M-\frac{m}{k} \theta_{b}\right) u,\left(b+M-2 \theta_{b}\right) v+\theta_{b}^{2} u\right)-\mathbf{u}, \\
& \mathbf{F}_{a, \mathbf{u}}\left(a_{1} ; \mathbf{v}^{*}\right) \mathbf{u}=\left((-\Delta+M)^{-1} u, 0\right) .
\end{aligned}
$$

易证, 0 是算子 $\mathbf{F}_{\mathbf{u}}\left(a_{1} ; \mathbf{v}^{*}\right)$ 的一个简单特征值, $\mathbf{u}_{0}=\left(u_{0}, v_{0}\right)$ 是对应的特征向量, 这里 $u_{0}$ 是对 应于特征值 $a_{1}=\lambda_{1}\left(\frac{m}{k} \theta_{b}\right)$ 的特征函数, $v_{0}=L_{b}^{-1}\left(\theta_{b}^{2} u_{0}\right)>0$, 算子 $L_{b}$ 由 (1.3) 式给出. 我们断 言: $\mathbf{F}_{a, \mathbf{u}}\left(a_{1} ; \mathbf{v}^{*}\right) \mathbf{u}_{0} \notin \mathcal{R}\left(\mathbf{F}_{\mathbf{u}}\left(a_{1} ; \mathbf{v}^{*}\right)\right)$. 事实上, 如果存在 $\mathbf{u}$, 使得 $\mathbf{F}_{a, \mathbf{u}}\left(a_{1} ; \mathbf{v}^{*}\right) \mathbf{u}_{0}=\mathbf{F}_{\mathbf{u}}\left(a_{1} ; \mathbf{v}^{*}\right) \mathbf{u}$, 则 $\Delta u-\frac{m \theta_{b}}{k} u+a_{1} u=u_{0}, x \in \Omega,\left.u\right|_{\partial \Omega}=0$. 由此推出 $\int_{\Omega} u_{0}^{2}=\int_{\Omega} u\left(\Delta u_{0}-\frac{m \theta_{b}}{k} u_{0}+a_{1} u_{0}\right)=0$, 这是一个矛盾.

根据局部分支理论 ${ }^{[16]},\left(a_{1}, \mathbf{v}^{*}\right)$ 是一个分支点. 并且在 $\left(a_{1}, \mathbf{v}^{*}\right)=\left(\lambda_{1}\left(\frac{m}{k} \theta_{b}\right), 0, \theta_{b}\right)$ 的邻 域内, 分支解可以写成

$$
(a, \mathbf{u})=(a(s), \mathbf{u}(s))=\left(a(s), s\left(u_{0}+\Phi(s)\right), \theta_{b}+s\left(v_{0}+\Psi(s)\right)\right), \quad|s| \leqslant s_{0},
$$


并且是问题 (1.1) 的唯一非平凡解, 这里 $0<s_{0} \ll 1, a(s), \Phi(s), \Psi(s) \in C^{1}$ 满足 $a(0)=$ $a_{1}, \Phi(0)=\Psi(0)=0, u_{0}>0$ 满足 $\int_{\Omega} u_{0}^{2}=1$. 显然, 当 $|s| \ll 1$ 时, 由 (3.1) 式给出的 $(a(s), \mathbf{u}(s))$ 是一个正解当且仅当 $s>0$. 利用定理 2.2 的 (i) 知, 当 $0<s \ll 1$ 时 $a(s)>a_{1}$. 这说明, 当 $s_{0} \ll 1$ 时, 在 $s \in\left[-s_{0}, 0\right)$ 上 $a(s)<a_{1}$.

把 $u(x)$ 写成 $u(x)=u(s ; x)=s\left(u_{0}(x)+\Phi^{\prime}(0, x) s+o(s)\right), 0<s \ll 1$. 因为 $u_{0}(x)>0$, $\left.\frac{\partial u_{0}}{\partial \nu}\right|_{\partial \Omega}<0$, 这里 $\nu$ 是 $\partial \Omega$ 上的单位外法向, 所以存在 $0<\bar{s} \ll 1$, 使得当 $x \in \Omega, 0<s \leqslant \bar{s}$ 时, $u(s ; x)>0$. 记 $u(s ; x)=u(a ; x), v(x)=v(a ; x), \mathbf{u}(x)=\mathbf{u}(a ; x)=(u(a ; x), v(a ; x))$. 上面的讨 论说明, 存在 $\bar{a}>a_{1}$, 使得当 $x \in \Omega, a_{1}<a \leqslant \bar{a}$ 时, $u(a ; x)>0$. 利用引理 3.1 , 我们有下面的 事实:

事实 $\mathbf{A}$ 不存在 $a^{*} \in\left(a_{1}, \infty\right)$, 使得 $\left.\mathbf{u}(a ; x)\right|_{\Omega}>0$ 对所有 $a_{1}<a<a^{*}$, 并且 $u\left(a^{*} ; x^{*}\right)=0$ 对某个 $x^{*} \in \Omega$ 成立. 这里, $\mathbf{u}(a ; x)>0$ 指 $u(a ; x)>0, v(a ; x)>0$.

设 $\mathcal{Z}$ 是包含分支点 $\left(a_{1}, \mathbf{v}^{*}\right)$ 的正解的连通分支, 定义

$$
a_{*}=\sup \left\{a>a_{1}:(t, \mathbf{u}) \in \mathcal{Z}, \mathbf{u}(t ; x)>0, \forall x \in \Omega, a_{1}<t \leqslant a\right\} .
$$

利用事实 $\mathrm{A}$ 知, 如果 $a_{*}<\infty$ 则 $u\left(a_{*} ; x\right)>0, x \in \Omega$. 由引理 1.1 知, $v\left(a_{*} ; x\right) \geqslant \theta_{b}(x)>0$ 在 $\Omega$ 内成立. 令

$$
\begin{array}{ll}
\mathcal{C}=\left\{(a, \mathbf{u}) \in \mathcal{Z}: a_{1} \leqslant a \leqslant a_{*}\right\} \backslash\left\{\left(a_{1}, \mathbf{v}^{*}\right)\right\}, & \text { 如果 } a_{*}<\infty, \\
\mathcal{C}=\left\{(a, \mathbf{u}) \in \mathcal{Z}: a_{1} \leqslant a<\infty\right\} \backslash\left\{\left(a_{1}, \mathbf{v}^{*}\right)\right\}, & \text { 如果 } a_{*}=\infty .
\end{array}
$$

因为 $\mathcal{Z}$ 是连通的, 问题 (1.1) 在 $\left(a_{1}, \mathbf{v}^{*}\right)$ 的邻域内有唯一非平凡解, 根据引理 3.1 和定理 2.2 的 (i), $\mathcal{C}$ 是连通的并且仅包含正解, 因此, $\mathcal{C}=\left.\left(\mathcal{Z} \backslash\left\{\left(a_{1}, \mathbf{v}^{*}\right)\right\}\right)\right|_{a \geqslant a_{1}}$.

下面证明 $a_{*}=\infty$, 从而定理的结论成立. 反证. 假设 $a_{*}<\infty$. 根据估计式 (1.4), $\mathcal{C}$ 是有 界的. 记 $\Lambda=a\left(-s_{0}\right)$, 则 $\Lambda<a_{1}$. 记 $\mathcal{C}_{\Lambda}=\{(a, \mathbf{u}) \in \mathcal{Z}: a \geqslant \Lambda\}$, 则 $\mathcal{C}_{\Lambda}=\left.\mathcal{C} \cup \mathcal{Z}\right|_{\Lambda \leqslant a \leqslant a_{1}}$ 是连 通和有界的. 我们将利用文献 [17] 的结果导出矛盾.

如果 $\lambda>0$ 是 $\mathbf{F}_{\mathbf{u}}\left(a ; \mathbf{v}^{*}\right)$ 的一个特征值且 $(u, v)$ 是对应的特征向量. 若 $u=0$, 则 $v \neq 0$ 并且满足

$$
-(1+\lambda) \Delta v+\lambda M v+\left(2 \theta_{b}-b\right) v=0,
$$

这说明 0 是算子 $-(1+\lambda) \Delta+\lambda M+2 \theta_{b}-b$ 的一个特征值. 由于算子 $-\Delta+2 \theta_{b}-b$ 的特征值 都是正的 ${ }^{[2,3]}$ 并且 $\lambda>0$, 此与特征值关于系数 $\lambda$ 的单调性相矛盾, 所以 $u \neq 0$, 并且满足

因此

$$
(1+\lambda) u=(-\Delta+M)^{-1}\left(a+M-\frac{m}{k} \theta_{b}\right) u \text {. }
$$

$$
\left\{\begin{array}{l}
-\Delta u+\frac{m \theta_{b}}{k} u+\frac{\lambda}{1+\lambda}\left(a+M-\frac{m \theta_{b}}{k}\right) u=a u, \quad x \in \Omega, \\
\left.u\right|_{\partial \Omega}=0, \quad u \neq \equiv, \quad x \in \Omega .
\end{array}\right.
$$

从而,

$$
a=\lambda_{j}\left(\frac{m \theta_{b}}{k}+\frac{\lambda}{1+\lambda}\left(a+M-\frac{m \theta_{b}}{k}\right)\right) \quad \text { 对某个 } j \geqslant 1 .
$$

反之, 如果存在正常数 $\lambda$ 和某个 $j \geqslant 1$, 使得 (3.3) 式成立, 则这个 $\lambda$ 是 $\mathbf{F}_{\mathbf{u}}\left(a ; \mathbf{v}^{*}\right)$ 的正特 征值, $\left(u^{*}, v^{*}\right)$ 是对应的特征向量, 这里的 $u^{*}$ 是 (3.2) 式对应于 $\lambda_{j}\left(\frac{m \theta_{b}}{k}+\frac{\lambda}{1+\lambda}\left(a+M-\frac{m \theta_{b}}{k}\right)\right)$ 
的特征函数, $v^{*}$ 是边值问题

$$
-(1+\lambda) \Delta v+\lambda M v+\left(2 \theta_{b}-b\right) v=\theta_{b}^{2} u, \quad x \in \Omega ;\left.\quad u\right|_{\partial \Omega}=0
$$

的解. 把 $\mathbf{F}(a ; \mathbf{u})$ 写成

$$
\mathbf{F}(a ; \mathbf{u})=\mathbf{F}_{\mathbf{u}}\left(a ; \mathbf{v}^{*}\right)\left(\mathbf{u}-\mathbf{v}^{*}\right)+\mathbf{H}\left(a ; \mathbf{u}-\mathbf{v}^{*}\right),
$$

其中 $\mathbf{H}(a ; \mathbf{w})$ 是 $\mathbf{w}$ 的高阶项. 若记 $\Sigma$ 是使得 $\mathbf{F}_{\mathbf{u}}\left(a ; \mathbf{v}^{*}\right)$ 不是同构的所有 $a$ 构成的集合, 则 $\Sigma=\left\{\lambda_{i}\left(\frac{m}{k} \theta_{b}\right)\right\}_{i=1}^{\infty}: \equiv\left\{a_{i}\right\}_{i=1}^{\infty}$. 定义区间

$$
J_{0}=\left(\Lambda, a_{1}\right), \quad J_{i}=\left(a_{i}, a_{i+1}\right), \quad i=1,2, \ldots
$$

记 $m_{j}$ 是 $\lambda_{j}\left(\frac{m \theta_{b}}{k}+\frac{\lambda}{1+\lambda}\left(a+M-\frac{m \theta_{b}}{k}\right)\right)$ 的代数重数. 对于任一固定的 $a \in J_{i}$, 我们断 言: 如果 $i=0$, 那么算子 $\mathbf{F}_{\mathbf{u}}\left(a ; \mathbf{v}^{*}\right)$ 没有正特征值, 如果 $i \geqslant 1$, 那么算子 $\mathbf{F}_{\mathbf{u}}\left(a ; \mathbf{v}^{*}\right)$ 正好有 $\sum_{\ell=1}^{i} m_{\ell}$ 个正特征值. 事实上, 如果定义

$$
g_{j}(\lambda)=\lambda_{j}\left(\frac{m \theta_{b}}{k}+\frac{\lambda}{1+\lambda}\left(a+M-\frac{m \theta_{b}}{k}\right)\right), \quad \lambda>0, \quad j \geqslant 1,
$$

则 $g_{j}(\lambda)$ 关于 $\lambda$ 是严格增的, 并且

$$
g_{j}(0)=\lambda_{j}\left(\frac{m \theta_{b}}{k}\right)=a_{j}, \quad g_{j}(\infty)=\lambda_{j}(a+M)=\lambda_{1}+a+M .
$$

当 $i=0$ 时. 由于 $a<a_{1}$, 利用 (3.4) 式容易看出, 对于任意的 $j \geqslant 1$, 不存在正数 $\lambda$, 使得 (3.3) 式成立. 因此, 算子 $\mathbf{F}_{\mathbf{u}}\left(a ; \mathbf{v}^{*}\right)$ 没有正特征值. 当 $i \geqslant 1$ 时, 对任意固定的 $j: 1 \leqslant j \leqslant i$, 因为 $a_{j} \leqslant a_{i}<a<g_{j}(\infty)$, 由 (3.4) 式推知, 存在唯一正数 $\lambda$, 使得 (3.3) 式对这样的 $j$ 成立. 对任意固定的 $j \geqslant i+1$, 因为 $a<a_{i+1} \leqslant a_{j}=g_{j}(0)$, 不存在正数 $\lambda$, 使得 (3.3) 式对这样的 $j$ 成立. 断言得证.

利用上述断言可得

$$
\left\{\begin{array}{l}
\operatorname{Ind}\left(\mathbf{F}_{\mathbf{u}}\left(a ; \mathbf{v}^{*}\right), \mathbf{0}\right)=1 \text { 如果 } a \in J_{0}, \\
\operatorname{Ind}\left(\mathbf{F}_{\mathbf{u}}\left(a ; \mathbf{v}^{*}\right), \mathbf{0}\right)=(-1)^{\sum_{\ell=1}^{i} m_{\ell}} \quad \text { 如果 } a \in J_{i}, i \geqslant 1 .
\end{array}\right.
$$

沿用文献 [15] 中的记号, 我们有

$$
P(1)=-1, \quad P(2)=\frac{1}{2}\left[1-(-1)^{m_{2}}\right], \quad P(i)=\frac{1}{2}(-1)^{\sum_{\ell=2}^{i-1} m_{\ell}}\left[1-(-1)^{m_{i}}\right], \quad \forall i \geqslant 3 .
$$

因为 $\mathcal{C}_{\Lambda}$ 有界, 根据文献 $\left[17\right.$, p. 753] 的二择一结果, 存在 $i \geqslant 2$, 使得 $P(i)=1$ 且 $\left(a_{i}, \mathbf{v}^{*}\right) \in \mathcal{C}_{\Lambda}$. 注意到 $a_{i}>a_{1}$, 有 $\left(a_{i}, \mathbf{v}^{*}\right) \in \mathcal{C}$. 此与 $\mathcal{C}$ 仅由正解构成的事实相矛盾. 证毕.

记 $k^{*}=\left(m \max _{\bar{\Omega}} \theta_{b}\right)^{1 / 2}$, 则 $k^{*}>\tilde{k}$, 这里的 $\tilde{k}$ 由定理 2.2 确定.

定理 3.2 (局部唯一性和稳定性) 设 $b>\lambda_{1}, k \geqslant k^{*}$, 则存在常数 $\delta=\delta(m, k, b)>0$, 使 得当 $a_{1}<a<a_{1}+\delta$ 时, 问题 (1.1) 有唯一正解并且是线性化稳定的, 其中 $a_{1}=\lambda_{1}\left(\frac{m}{k} \theta_{b}\right)$.

证明 存在性由定理 3.1 给出. 为证唯一性, 从定理 3.1 的证明容易看出, 只需证明对 每个满足 $a_{i} \rightarrow a_{1}$ 的序列 $\left\{a_{i}\right\}$, 问题 (1.1) 对应于 $a=a_{i}$ 的正解 $\left(u_{i}, v_{i}\right)$ 在 $Z$ 中收玫于 $\left(0, \theta_{b}\right)=\mathbf{v}^{*}$. 反证. 假设存在 $\left\{\left(u_{i}, v_{i}\right)\right\}$ 的一个子列, 仍记为 $\left\{\left(u_{i}, v_{i}\right)\right\}$, 使得 $\left(u_{i}, v_{i}\right)$ 在 $Z$ 中收 敛于 $(\hat{u}, \hat{v})$ 并且 $(\hat{u}, \hat{v}) \neq\left(0, \theta_{b}\right)$, 则 $(\hat{u}, \hat{v})$ 是问题

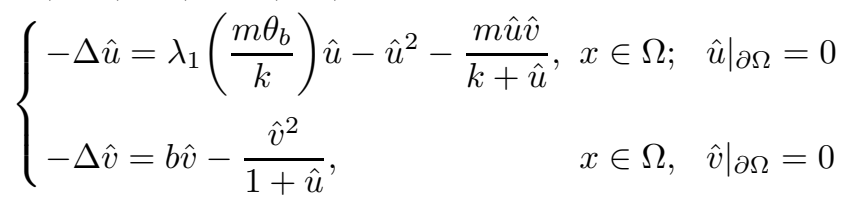


的一个非负解. 如果 $\hat{v}=0$, 则 $v_{i} \rightarrow 0$ 在 $\bar{\Omega}$ 上一致成立. 令 $\tilde{v}_{i}=\frac{v_{i}}{\left\|v_{i}\right\|_{\infty}}$, 则 $\tilde{v}_{i} \rightarrow \tilde{v}$, 并且 $\tilde{v}$ 是 问题

$$
-\Delta \tilde{v}=b \tilde{v}, \quad x \in \Omega,\left.\quad \tilde{v}\right|_{\partial \Omega}=0
$$

的一个非平凡的非负解. 由于 $b>\lambda_{1}$, 这是不可能的. 如果 $\hat{v} \neq 0, \hat{u}=0$, 那么 $\hat{v}=\theta_{b}$. 此与 $(\hat{u}, \hat{v}) \neq\left(0, \theta_{b}\right)$ 矛盾. 如果 $(\hat{u}, \hat{v})$ 是 $(3.5)$ 式的正解, 则 $(\hat{u}, \hat{v})$ 是问题 (1.1) 对应于 $a=a_{1}=\lambda_{1}\left(\frac{m}{k} \theta_{b}\right)$ 的正解. 因为 $k>\tilde{k}$, 此与定理 2.2 矛盾.

下面证明, 当 $a_{1}<a<a_{1}+\delta$ 时 (如有必要, 把 $\delta$ 取得更小), 由定理 3.1 得到的问 题 $(1.1)$ 的唯一正解 $\mathbf{u}(s)=(u(s), v(s))$ 是稳定的. 等价地, 只要证明当 $0<s \ll 1$ 时, $(u(s), v(s))$ 是稳定的. 我们将利用线性化理论来证明 [18,19]. 设 $\mathcal{F}(a ; \mathbf{u})$ 由 $(2.3)$ 式定义. 容 易看出 $\mathcal{F}_{\mathbf{u}}\left(a ; 0, \theta_{b}\right): X \cap Z \rightarrow Y$ 是连续的并且 0 是 $\mathcal{F}_{\mathbf{u}}\left(a_{1} ; 0, \theta_{b}\right)$ 的一个 $I$-简单特征值 ${ }^{[16,19] \text {, }}$ 其中 $a_{1}=\lambda_{1}\left(\frac{m}{k} \theta_{b}\right)$. 根据线性化稳定性理论 ${ }^{[18,19]}$, 在 $\left(a_{1}, 0, \theta_{b}\right)$ 的小邻域内, 存在两个连续 可微函数 $a \rightarrow(\mu(a), \mathbf{w}(a))$ 和 $s \rightarrow(\beta(s), \mathbf{z}(s))$, 分别定义在 $a_{1}$ 和 0 的邻域内, 其值域属于 $R \times Z$, 并且满足

$$
\begin{aligned}
& \mu\left(a_{1}\right)=\beta(0)=0, \quad \mathbf{w}\left(a_{1}\right)=\mathbf{z}(0)=\left(u_{0}, v_{0}\right), \\
& \mathcal{F}_{\mathbf{u}}\left(a ; 0, \theta_{b}\right) \mathbf{w}(a)=\mu(a) \mathbf{w}(a), \text { 当 }\left|a-a_{1}\right| \ll 1, \\
& \mathcal{F}_{\mathbf{u}}(a(s) ; u(s), v(s)) \mathbf{z}(s)=\beta(s) \mathbf{z}(s), \text { 当 }|s| \ll 1 .
\end{aligned}
$$

同时, $\mu^{\prime}\left(a_{1}\right) \neq 0$, 并且 $s a^{\prime}(s) \mu^{\prime}\left(a_{1}\right)$ 与 $\beta(s)$ 反号. 为证明 $(u(s), v(s))$ 的稳定性, 必须确 定 $\beta(s)$ 的符号 $(0<s \ll 1)$. 为此目的, 我们先确定 $a^{\prime}(s)$ 和 $\mu^{\prime}\left(a_{1}\right)$ 的符号. 根据 RieszSchauder 理论 ${ }^{[15]}, \sigma\left(\mathcal{F}_{\mathbf{u}}\left(a ; 0, \theta_{b}\right)\right)=\sigma\left(\Delta+a-\frac{m}{k} \theta_{b}\right) \cup \sigma\left(-L_{b}\right)$, 其中 $\sigma(T)$ 表示算子 $T$ 的谱. 记 $\mathbf{w}(a)=\left(w_{1}(a), w_{2}(a)\right)$. 简单计算知

$$
\Delta w_{1}(a)+\left(a-\frac{m}{k} \theta_{b}\right) w_{1}(a)=\mu(a) w_{1}(a), \quad x \in \Omega ;\left.\quad w_{1}(a)\right|_{\partial \Omega}=0 .
$$

因为 $u_{0}$ 是 $C_{0}^{+}(\bar{\Omega}) \equiv\left\{u \in C_{0}(\bar{\Omega}):\left.u\right|_{\Omega} \geqslant 0\right\}$ 的内点, 所以当 $\left|a-a_{1}\right| \ll 1$ 时, 在 $\Omega$ 内 $w_{1}(a)>0$. 因此当 $\left|a-a_{1}\right| \ll 1$ 时, $\mu(a)=a-\lambda_{1}\left(\frac{m \theta_{b}}{k}\right)=a-a_{1}$, 从而 $\mu^{\prime}\left(a_{1}\right)=1$. 另一方面, 把 $(a(s), u(s), v(s))$ 代入问题 (1.1) 的第 1 个方程并关于 $s$ 微分, 再取 $s=0$, 可得

$$
\left\{\begin{array}{l}
\Delta \Phi^{\prime}(0)=\left(\frac{m \theta_{b}}{k}-\lambda_{1}\left(\frac{m \theta_{b}}{k}\right)\right) \Phi^{\prime}(0)-a^{\prime}(0) u_{0}+u_{0}^{2}+m \frac{k v_{0}-\theta_{b} u_{0}}{k^{2}} u_{0}, \quad x \in \Omega, \\
\Phi^{\prime}(0)=0, \quad x \in \partial \Omega .
\end{array}\right.
$$

用 $u_{0}$ 乘以 (3.6) 式并积分, 再利用 $u_{0}$ 的定义, 有

$$
a^{\prime}(0)=\frac{m}{k} \int_{\Omega} v_{0} u_{0}^{2} \mathrm{~d} x+\int_{\Omega}\left(1-\frac{m}{k^{2}} \theta_{b}\right) u_{0}^{3} \mathrm{~d} x .
$$

由于 $k \geqslant k^{*}$, 故 $1-\frac{m}{k} \theta_{b}^{2}(x)>0, x \in \Omega$. 因为 $u_{0}, v_{0}>0$, 所以 $a^{\prime}(0)>0$. 因此 $\beta(s)<0$, $0<s \ll 1$. 由此推出当 $a_{1}<a<a_{1}+\delta$ 时, 问题 (1.1) 的正解 $(u(s), v(s))$ 是稳定的. 证毕.

定理 3.3 设 $a, b>\lambda_{1}$. 如果 $0<m \ll 1$ 或者 $k \gg 1$, 那么问题 (1.1) 有唯一正解 $\mathbf{u}$ 并 且是线性化稳定的.

证明 只证 $m$ 很小的情形. 先证唯一性, 其基本方法是隐函数定理. 记 $\mathbf{u}_{a}=\left(\theta_{a}, \theta_{a, b}\right)$ 由第 1 节给出, 并把 $\mathcal{F}(a ; \mathbf{u})$ 写成 $\mathbb{F}(m ; \mathbf{u})$, 见 $(2.3)$ 式, 则 $\mathbf{u}_{a}$ 是 $\mathbb{F}(0 ; \mathbf{u})=0$ 的唯一正解. 简 
单计算知

$$
\mathbb{F}_{\mathbf{u}}\left(0 ; \mathbf{u}_{a}\right)=\left(\Delta u+\left(a-2 \theta_{a}\right) u, \Delta v+\left(b-\frac{2 \theta_{a, b}}{1+\theta_{a}}\right) v+\frac{\theta_{a, b}^{2}}{\left(1+\theta_{a}\right)^{2}} u\right) .
$$

同于定理 2.2 的证明, $\mathbb{F}_{\mathbf{u}}\left(0 ; \mathbf{u}_{a}\right)$ 是满单射. 根据隐函数定理, 存在正常数 $m_{0}$ 和 $\rho$, 使得 对每一个 $m \in\left[0, m_{0}\right)$, 方程 $\mathbb{F}(m ; \mathbf{u})=0$ 落在 $B_{\rho}\left(\mathbf{u}_{a}\right)$ 内的解是唯一的. 为证明对每一个 $m \in\left[0, m_{0}\right)$, 方程 $\mathbb{F}(m ; \mathbf{u})=0$ 的解是唯一的, 我们需要证明当 $m \rightarrow 0^{+}$时, 对应的正解 $\mathbf{u} \rightarrow \mathbf{u}_{a}$. 事实上, 由椭圆型方程的正则性理论知, $\mathbf{u} \rightarrow \tilde{\mathbf{u}}=(\tilde{u}, \tilde{v})$. 因为当 $m \rightarrow 0$ 时 $\frac{m u v}{k+u} \rightarrow 0$, 所以 $\mathbb{F}(0, \tilde{\mathbf{u}})=0$. 又因为 $a>\lambda_{1}$, 故 $\tilde{u}=\theta_{a}, \tilde{v}=\theta_{a, b}$.

再证稳定性. 容易看出当 $m \rightarrow 0$ 时, 问题 (1.1) 是下面的问题

$$
\left\{\begin{array}{l}
-\Delta u=a u-u^{2}, \quad x \in \Omega,\left.u\right|_{\partial \Omega}=0, \\
-\Delta v=b v-\frac{v^{2}}{1+\theta_{a}}, \quad x \in \Omega,\left.v\right|_{\partial \Omega}=0
\end{array}\right.
$$

的一个摄动. 因为 $\mathbf{u}_{a}=\left(\theta_{a}, \theta_{a, b}\right)$ 是问题 (3.7) 的唯一正解且是稳定的, 根据线性算子的摄动 理论 [20], 当 $0<m \ll 1, a, b>\lambda_{1}$ 时, 问题 (1.1) 的正解是线性稳定的. 证毕.

注 3.1 固定 $a, b, k$ 且 $a, b>\lambda_{1}$. 考虑 $m$ 作为分支参数. 注意到 $f(m) \equiv \lambda_{1}\left(\frac{m}{k} \theta_{b}\right)$ 是 $m$ 的严格增函数, $f(0)=\lambda_{1}$ 并且 $f(\infty)=\infty$, 故存在唯一的 $m_{0}=m(a)$, 使得 $a=\lambda_{1}\left(m_{0} \theta_{b} / k\right)$. 同于定理 3.1 和 3.2 , 并利用定理 3.3 可以推出, 在 $m$ - $\mathbf{u}$ 平面存在问题 (1.1) 的正解的连通分 支 $\mathcal{C}_{m}$ 连接 $\left(m_{0}, \mathbf{v}^{*}\right)$ 和 $\left(0, \mathbf{u}_{a}\right)$, 并且当 $0<m_{0}-m \ll 1$ 或者 $0<m \ll 1$ 时, $\mathbf{u}(m)$ 是唯一 的、线性稳定的.

注 3.2 如果固定 $a, b, m$ 满足 $a, b>\lambda_{1}$ 并考虑 $k$ 为分支参数. 取 $k_{0}$ 由 $a=\lambda_{1}\left(\frac{m}{k_{0}} \theta_{b}\right)$ 唯 一确定. 同上可得, 在 $k-\mathbf{u}$ 平面存在问题 (1.1) 的正解的连通分支 $\mathcal{C}_{k}$ 连接 $\left(k_{0}, \mathbf{v}^{*}\right)$ 和 $\left(\infty, \mathbf{u}_{a}\right)$, 并且当 $0<k-k_{0} \ll 1$ 或者 $k \gg 1$ 时, $\mathbf{u}(k)$ 是唯一的、线性稳定的.

注 3.3 固定 $a, m, k$ 满足 $a>\lambda_{1}$ 并考虑 $b$ 是分支参数. 注意到注 2.1 , 同于定理 3.1和 3.2 , 可证 $\left(\lambda_{1}, \mathbf{u}^{*}\right)$ 是一个分支点, 存在问题 (1.1) 的正解的连通分支 $\mathcal{C}_{b}(s)=\{(b(s), \mathbf{u}(s)): 0<s<$ $\left.s_{0}\right\}$, 使得 $b(0)=\lambda_{1}, \mathbf{u}(0)=\mathbf{u}^{*}=\left(\theta_{a}, 0\right), b\left(s_{0}\right) \in\left(\lambda_{1}, \infty\right)$. 同时存在常数 $\delta=\delta(a, m, k)>0$, 使 得当 $\lambda_{1}<b<\lambda_{1}+\delta$ 时, 问题 (1.1) 的正解是唯一的、线性稳定的.

\section{致谢感谢审稿人提出的宝贵修改建议.}

\section{参考文献}

1 Aziz-Alaoui M A, Okiye M D. Boundedness and global stability for a predator-prey model with modified Leslie-Gower and Holling-Type II Schemes. Appl Math Lett, 16: 1069-1075 (2003)

2 Blat J, Brown K J. Global bifurcation on positive solutions in some systems of elliptic equations. SIAM J Math Anal, 17: 1339-1353 (1986)

3 Yamada Y. Stability of steady states for prey-predator diffusion equations with homogeneous Dirichlet conditions. SIAM J Math Anal, 21: 327-345 (1990)

4 Ruan W, Feng W. On the fixed point index and multiple steady states of reaction-diffusion systems. Differential Integral Equations, 8: 371-391 (1995)

5 Alves C O, de Figueiredo D G. Nonvariational elliptic systems. Discrete Contin Dyn Syst, 8(2): 289-302 $(2002)$ 
6 Amann H. Fixed point equations and nonlinear eigenvalue problems in ordered Banach spaces. SIAM Review, 18: 620-709 (1976)

7 Dancer E N. On the indices of fixed ponits of mappings in cones and applications. J Math Anal Appl, 91(1): 131-151 (1983)

8 Dancer E N, Du Y H. Effects of certain degeneracies in the predator-prey model. SIAM J Math Anal, 34: 292-314 (2002)

9 Delgado M, López-Gómez J. On the symbiotic Lotka-Volterra model with diffusion and transport effects. J Differential Equations, 160: 175-262 (2000)

10 Du Y H, Lou Y. Some uniqueness and exact multiplicity results for a predator-prey model. Trans Amer Math Soc, 349(6): 2442-2475 (1997)

11 Du Y H, Lou Y. S-shaped global bifurcation curve and Hopf bifurcation of positive solutions to a predatorprey model. J Differential Equations, 144: 390-440 (1998)

12 Gui C F, Lou Y. Uniqueness and nonuniqueness of coexistence states in the Lotka-Volterra competition model. Commun Pure Appl Math, XLVII: 1571-1594 (1994)

13 Li L. Coexistence theorems of steady-states for predator-prey interacting systems. Trans Amer Math Soc, 305(1): 143-166 (1988)

14 Ruan W, Pao C V. Positive steady-state solutions of a competing reaction-diffusion system. J Differential Equations, 117: 411-427 (1995)

15 Wang M X. Nonlinear Partial Differential Equations of Parabolic Type (in Chinese). Beijing: Science Press, 1993

16 Crandall M G, Rabinowitz P H. Bifurcation from simple eigenvalues. J Funct Anal, 8: 321-340 (1971)

17 López-Gómez L, Mora-Corral C. Minimal complexity of semi-bounded components in bifurcation theory. Nonlinear Anal, 58: 749-777 (2004)

18 Crandall M G, Rabinowitz P H. Bifurcation, perturbation of simple eigenvalues, and linearized stability. Arch Ration Mech Anal, 52(1): 161-180 (1973)

19 Nirenberg L. Topics in Nonlinear Functional Analysis. Providence: American Mathematical Society, 2001

20 Kato K. Perturbation Theory for Linear Operator. New York: Springer-Verlag, 1966 